\title{
Bulk SMS Communication Portal
}

\author{
Nishanthi $\mathrm{G}^{\mathrm{a}, 1}$, and VimalaS ${ }^{\mathrm{b}}$ \\ ${ }^{a} P G$ Scholar, Dept of CSE, Panimalar Engineering College, Chennai \\ ${ }^{b}$ Associate Professor, Dept of CSE, Panimalar Engineering College, Chennai
}

\begin{abstract}
Sending SMS to large amount of people in an organization is a tedious process and it requires more human effort tocomplete the task. The best solution in this aspect could be developing a portal which facilitate sending bulk SMS to multiple users . But the problem here is the portal should be more user-friendly. Otherwise, the portal also requires more human effort in entering data like mobile number for large amount of people. This project provide a solution for organization to send bulk SMS and with various features like log generation, notification of failure ofSMS and/or delivery, scheduling of SMS,alert/notification system,read and unread status, provision of importing contact details from excel worksheets, customizing with name of the receiver and so on. The application can be easily used and maintained by an administrator in the organization in a more effective manner.
\end{abstract}

Keywords. SMS,bulkmessages,PCP

\section{Introduction}

Bulk message sending through multiple members while sending SMS messages transport to smart phone terminals. It is utilized by media organizations, banks (for advertising and marketing) and client brands for a diffusion of functions such as entertainment, corporation and mobile advertising and marketing places bulk messaging is commonly used for marketing. But additionally for statistics and communication among both group of workers and customers. This task offer a solution for company to send bulk SMS and with numerous capabilities like log details, notification of failure of SMS and/or delivery, scheduling of SMS, alert/notification system, read and unread status, provision of uploading touch info from excel worksheets, customizing with name of the receiver and so on.

\section{Literature Survey}

In this paper[1] has shown that longer electronic mail addresses and uncovered new person behaviors in continually-on ubiquitous international. The bulk mail threats, the mobile electronic mail deal with expands its length and unpredictability. The server is very safety by using this anti-ube when compare from electronic mail.[2]bulk message delivered community-wide congestion gray-container telephony and voip and buffer technique sending a message thru a networks extensive congestion happens. The short message service has been the most famous and profitable huge-place wi-fi data provider. [3] .SMS sender can skip the captcha check, it will likely be identified that SMS is legitimate text to speech method, MMS .Here drawbacks is run on a normal

\footnotetext{
${ }^{1}$ Nishanthi G, PG Scholar, Dept of CSE ,Panimalar Engineering College, Chennai, India;

E-mail:nishanishanthi3791@gmail.com
} 
mobile phone due to loss of any complex method. In this paper[4] the highest entropy first with out lacking any real time scheduling to use entropy in actual-time scheduling, the set of rules' $\mathrm{s}$ goal is to reduce the uncertainty of the system by scheduling the venture with the best entropy first.In this paper[5] time cloud sources are underutilized due to negative scheduling of assignment (or utility) in datacenter.vm drawbacks QOS reduce the value with out violating the SLA (service level agreement) for an software in cloud SAAS environment.

\section{Proposed system}

The actual-time system depends at the logical result however additionally at the time also to be measured.There is a scheduling algorithm taken used in this algorithm identified the difficulty of multi-challenge the given time to be optimized.The wide variety of servers and the performance indicator in actual-time system that is PCP Scheduling algorithm. The best solution in this aspect could be developing a portal which facilitate sending bulk SMS to multiple users.But the problem here is the portal should be more user-friendly.The scheduling resources, and the scheduling of actualtime assignment emphasizes time constraint.Actual-time structures are defined as those systems in which the correctness of the system depends no longer most effective at the logical result of computation, however also on the time at which the consequences are produced.

\subsection{Algorithm}

PCP planning pseudo code

\section{Algorithm 1: The PCP planning Algorithm}

- The scheduling work flow methods is applied Here, is SCHEDULE WORKFLOWG(G(T,E),D)

○ select the services assign for each task $\mathrm{G}$.

o From $t$ entry, $t$ exit and their corresponding edges to $G$

○ Assign MET(T i ) for edge

- Assign MTT(e (i,j )) for each edge

- Assign EST(T,i ) for each task in $\mathrm{G}$

- Assign LFT(T,i ) for each task in $\mathrm{G}$

- Sub-deadline(T start ) 0, sub-deadline(T End ) D

○ start from ' $\mathrm{T}$ ' start and ' $\mathrm{T}$ ' end as assigned

- Assign Parents(T End)

$\circ$ process services are assigned $(\mathrm{G}(\mathrm{T}, \mathrm{E}))$

$\circ$ End process

\section{Module Description}

\subsection{Communication Portal Interface Design}

This module is the user interface design for the user to access the Bulk SMS Communication Portal. It consists of user registration and user login.After registration 
and login, there is option to create or choose contents to send SMS to multiple users. The user password is retrieved by using this portal. In user registration module user enters the user name. When client entered the all user details in registration completed, the user registration data is stored in data base collecting the information is base on utilized during login stage for confirmation.

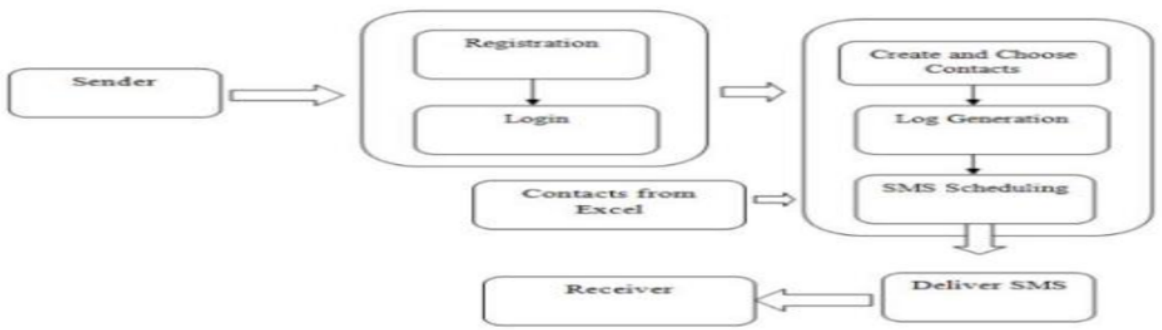

Figure 1. Architecture diagram

\subsection{Log Generation}

Text Later is $\log$ generation function that permits Your texts at a later time and date. Everything that you do inner portal is logged proper on your inventory SMS portal. So if you ever need to discover a message which you sent the usage of this app, it available for your stock messaging portal. The essential display of the portal offers to schedule a brand new SMS or view the ones that you have already scheduled.

\subsection{SMS Scheduling}

SMS Scheduling is an automated function which sends the text messages you written after a certain period of time with certain contents. Just type a message and save it. Choose an SMS scheduling and set the date and time When you need to send the message, Without no similarly problem from you, the portal will send your stored message at the exact date With the capability of helping you by sending instant messages at a preset time, the application does the ideal occupation of booking SMS for you. It has a lovely and disorder free interface from where you can deal with your booked messages. This feature is perfect for when you know you will not be at your desk when an SMS message needs to you can schedule your SMS text messages to be sent at a future date and time - up to a year into the future.

\subsection{SMS Delivery}

The scheduled SMS is sent or deliver to the recipient through this module. It also shows a report which says that message has been sent from this device. It does not means the SMS has been delivered to the receiver. Yes, Once we click the send SMS in mobile or system, it get decodes into binary form and passes through the air medium.Finally it reaches to the SMSC( Short Message Service Center), there it gets stored and it releases the message to particular receiver or operator according to the availability of its network. When you send an SMS, it is first added to an SMS message centre. It is then introduced from message centre to meant recipient's mobile 
phone if it is able to be located on community.

\section{Result}

Here we compare our proposed PCP algorithm with existing methods, Bayesian Network and Random Forest Algorithm.

\subsection{Accuracy}

Precision is resolved as the general model is registered and absolute genuine characterization parameters $\left(\mathrm{T} p+\mathrm{T}_{\mathrm{n}}\right)$ which is segregated by the sum of the classification parameters $\left(T_{p}+T_{n}+F_{p}+F_{n}\right)$.

The accuracy is computed as like :

$$
\text { Accuracy }=\frac{T_{p}+T_{n}}{\left(T_{p}+T_{n}+F_{p}+F_{n}\right)}
$$

Where $_{\mathrm{p}^{-}}$True positive,

$\mathrm{F}_{\mathrm{n}}$-False (-ve)

$\mathrm{F}_{\mathrm{p}}$-False (+ve)

Figure 2,the given table to be observed that the contrast metric is evaluated using current and proposed method in phrases of accuracy.

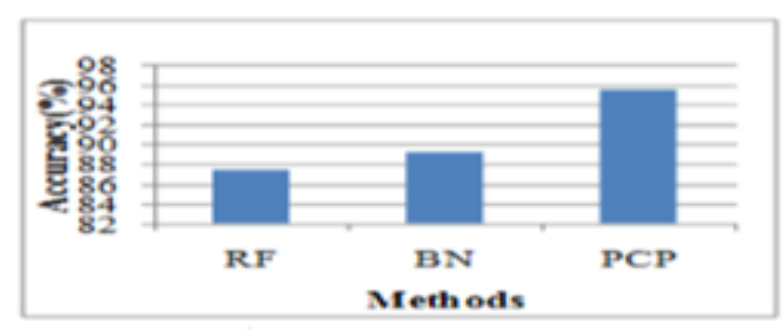

Figure 2. Accuracy comparison

\subsection{Precision}

Precision is discusses about the ratio of the positives or both (+) positives and (-) positives result for imposition and real features. It is as given below

$$
\text { Precision }=\frac{\{\text { Relevent document }\} \&\{\text { Retrieved document }\}}{\mid\{\text { Retrieved document }\} \mid}
$$




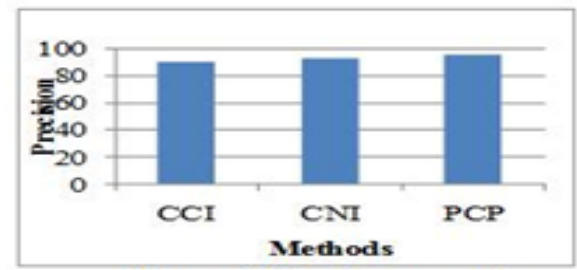

Figure 3. precision comparison

The above given Figure 3.in this metric method evaluated using existing and proposed method identified precision.

\section{Conclusion}

Thus the web application for sending bulk SMS will be definitely helpful for organizations which are in need of these kind of applications this software program development that is primarily based on brief messaging provider (SMS)machine for handing over messages via SMS gateway. This system is most beneficial and makes use of SMS gateway which is emerging generation utilized by different advertising and marketing and notifications provider company like super market, colleges, weather forecasting facilities. This software program became designed based on usual realistic packages. From the diverse exams achieved, the designed software became located to be dependable and realistic. More capabilities may be brought from the prototype layout . Specifically, the database had additionally been examined using a few units of facts and it had been found to be successful. However, it has been tested the use of actual phone

\section{References}

[1] Riordan, B., \&amp; Traxler, J. (n.d.). The Use of Targeted Bulk SMS Texting to Enhance Student Support, Inclusion and Retention. IEEE International Workshop on Wireless and Mobile Technologies in Education (WMTE'05). doi:10.1109/wmte.2005.65

[2] Starsky H.Y. Wong, Songwu Lu (2007).Analysis of the reliability of Nationwide short message services.IEEE Communications Society subject matter experts for publication in the IEEE INFOCOM 2007 proceedings.

[3] Starsky H.Y. Wong, Songwu Lu (2007).Analysis of the reliability of Nationwide short message services.IEEE Communications Society subject matter experts for publication in the IEEE INFOCOM 2007 proceedings.

[4] RealTimeSystemsSymposium.doi:10.1109/rtss.2015.44.C., C. A. R., \& Cheng, A. M. K. (2015). Using Entropy as a Parameter to Schedule Real-Time Tasks.

[5] Lakra, A. V., \&amp; Yadav, D. K. (2015). Multi-Objective Tasks Scheduling Algorithm for Cloud Computing Throughput Optimization. Procedia Computer Science, 48, 107-113. doi:10.1016/j.procs.2015.04.158 